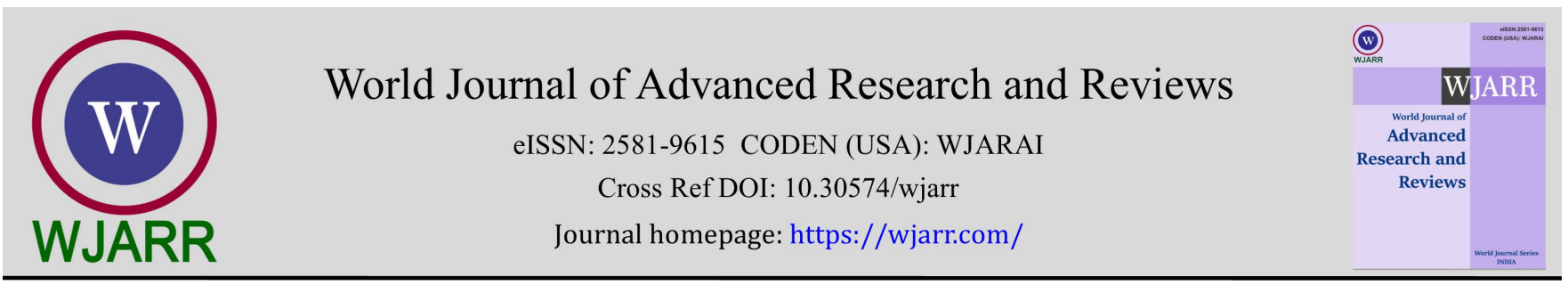

(CASE REPORT)

Check for updates

\title{
Rare presentation of incomplete male urethral duplication: A case report and review of literature
}

Orgeness J Mbwambo 1, 2,*, Alex Mremi ${ }^{3}$, Mohamed Mbarouk 1 , Jasper Mbwambo 1, 2, Frank Bright 1, 2, Alfred K Mteta ${ }^{1,2}$ and Bartholomeo Nicholaus Ngowi 1,2

\author{
${ }^{1}$ Department of Urology, Faculty of Medicine. Kilimanjaro Christian Medical University College. P.O. Box 2240. Moshi, \\ Tanzania \\ 2 Department of Urology, Kilimanjaro Christian Medical Centre, P.O. Box 3010, Moshi, Tanzania. \\ ${ }^{3}$ Department of Pathology. Kilimanjaro Christian Medical Centre. P.O. Box 3010, Moshi, Tanzania. \\ 4 Department of Pathology, Kilimanjaro Christian Medical University College, P.O. Box 2240, Moshi, Tanzania.
}

World Journal of Advanced Research and Reviews, 2021, 09(01), 292-296

Publication history: Received on 11 January 2021; revised on 20 January 2021; accepted on 22 January 2021

Article DOI: https://doi.org/10.30574/wjarr.2021.9.1.0017

\begin{abstract}
Urethral duplication is a rare congenital anomaly affecting mainly males. Here, we report a case of urethral duplication in a 19 years old male presented as a scrotal sinus discharging pus for 1 year. Surgical removal of accessory urethra was done and postoperative period was uneventful.
\end{abstract}

Keywords: Urethral duplication; Kilimanjaro Christian Medical University College.

\section{Background}

Urethral duplication is a very rare congenital anomaly with variable clinical presentations that can occur early or late in life [1]. Herein, we report our experience of late presentation of urethral duplication that presented as a scrotal sinus for one year in a 19-year-old male. The fistulogram and anterograde urethrogram were used to plan the management of the patient. The malformation was Effman Type 2A "Y type" and excision of accessory urethra was done successfully.

Purpose of the study

The purpose of this case report is to share our experience of management of a rare case of urethral duplication with late presentation in a teenager.

\section{Case report}

A 19 years old male presented with a history of recurrent foul smelling pus discharge on the scrotum opening for 1 year. However, there was no history of lower urinary tract symptoms, dysuria or being treated for urinary tract infections, double stream, abdominal pain, perineal trauma, fever, excessive night sweat or loss of weight.

He had undergone sinus excision with the diagnosis of pilonidal sinus 6 months prior to admission to our health facility. However, pus discharge recurred 1 month after the surgery.

\footnotetext{
* Corresponding author: Orgeness J Mbwambo

Department of Urology, Faculty of Medicine. Kilimanjaro Christian Medical University College. P.O. Box 2240. Moshi, Tanzania.

Copyright $(2021$ Author(s) retain the copyright of this article. This article is published under the terms of the Creative Commons Attribution Liscense 4.0.
} 
On physical examination he had normal male external genitalia with bilateral; palpable testis in the scrotum and external urethral meatus at the tip of glans penis. A small midline scrotal opening was identified and upon squeezing, a milky coloured fluid comes out of it. The fluid cultured Escheria coli that was $100 \%$ sensitive to Amikacin and Meropenem and $100 \%$ resistance to gentamicin, ceftriaxone and ciprofloxacin. Ultrasound of the urinary tract was normal.

A fistulogramme demonstrated a small irregular tract starting from scrotum to the level of bulbar urethra (figure 1). Micturating cystourethrogramme was normal with no vesicoureteric reflux or obvious contrast going to the accessory urethra. A provisional diagnosis of double urethra was reached (Effman type IIA2 "Y type") and the patient was planned for urethrocystoscopy and sinus exploration on the same sitting.

During urethrocystoscopy through the external urethral meatus that was located at the tip of penis, there was no obvious communication even after feeling the sinus tract with methylene blue. However, there was obvious lineal distension/bulging throughout the perineum (figure 3).

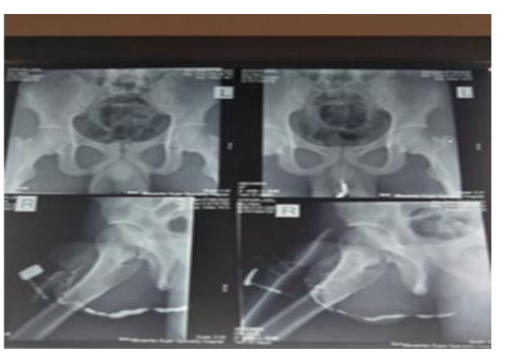

Figure 1 Fistulogram showing contrast in accessory urethral

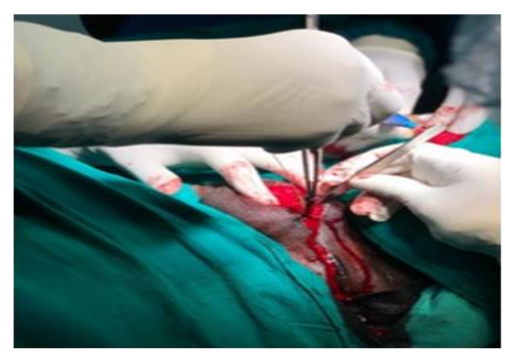

Figure $4 \mathrm{~A}$ : stillet inside ectopic urethra B: ectopic urethral C: methylene blue in the urethra

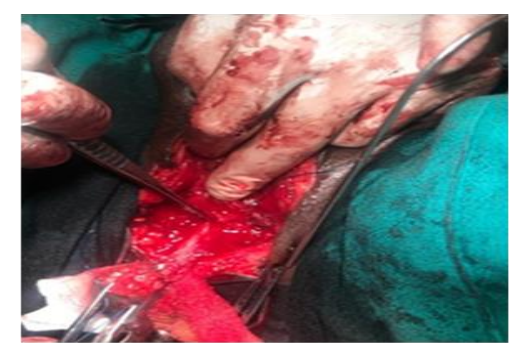

Figure 7 A: end of accessory urethra at proximal bulbar urethra, spongiosum opened but intact mucosa

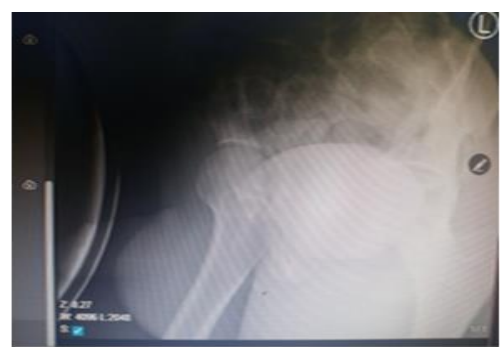

Figure 2 Antegrade urethrogram, no contrast in accessory urethra: obliterated junction between native and accessory

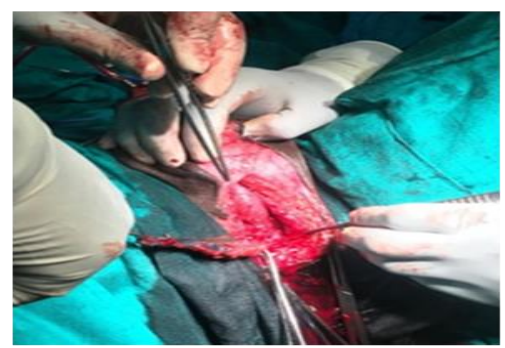

Figure 5 A: Bulbar urethra B ectopic urethral going into proximal bulbar urethra

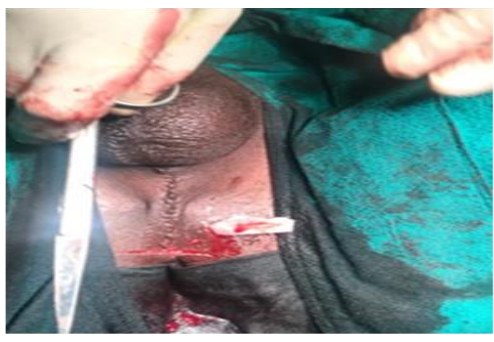

Figure 8 Wound closing with modified penrose drain in situ (Arrow)

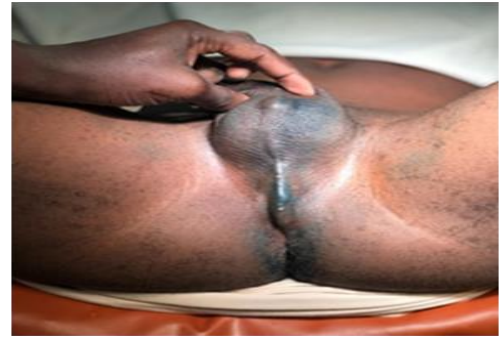

Figure 3 A: Accessory urethra $B$ : Methyleneblue in ectopic channel of urethra before surgeon

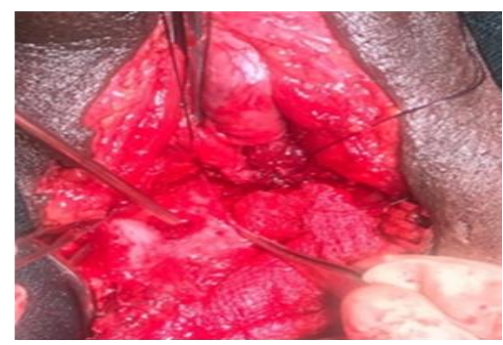

Figure 6 A: bulbar urethra with intact spongiosum above the sinus tract $B$ : Bulbar urethra with spongiosum opened following the sinus tract C: proximal part of sinus tract with well epithelialized mucosa

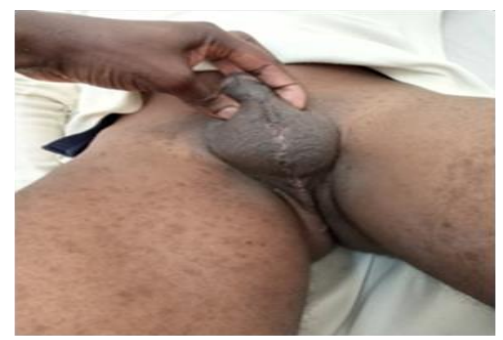

Figure 9 Healing wound 2 weeks after surgery 

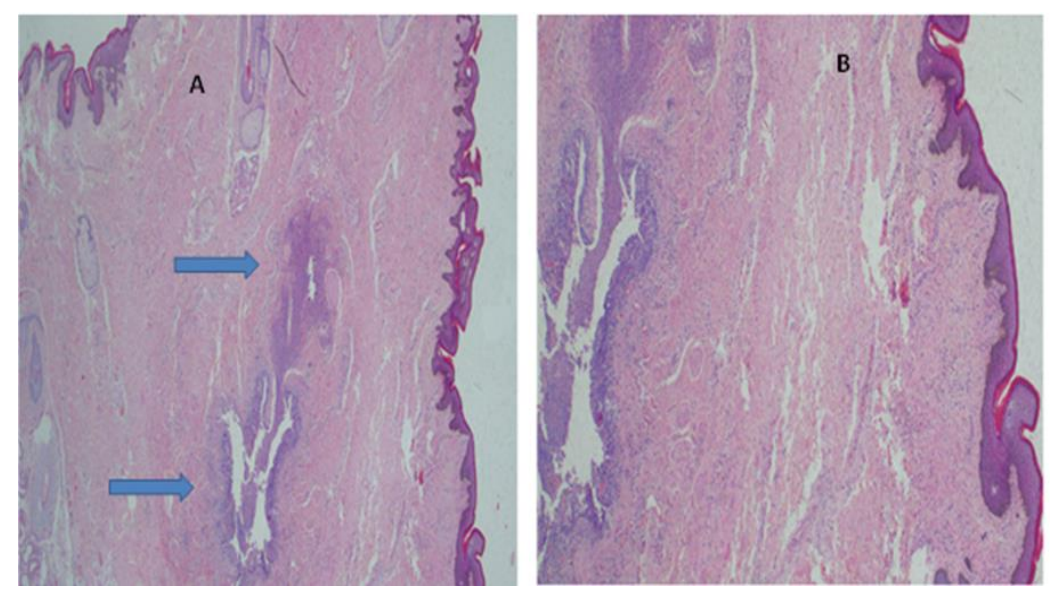

Figure 10 Photomicroscopy demonstrating sinus tract lined with epithelium and mixed acute and chronic inflammation, Hematoxylin and Eosine x 20 (A) and x 40 (B)

Surgical removal of sinus (figure 4-7) was done through median raphe perineal incision that extended from where the sinus opening was (mid scrotal area) up to $2 \mathrm{~cm}$ from the anal opening. The tract was identified at the scrotal area and followed down up to the level of proximal bulbous urethral where it was entering the corpus spongiousm. Spongiosum was opened, sinus tract freed from the spongiosum without opening the urethral mucosae. The blind ending proximal part of the sinus tract was ligated by vicrly 3, 0 just on the edge of mucosa of proximal bulbous urethra, excision accomplished. Corpus spongiosum was repaired with vicryl 4, 0, (figure 8). The final diagnosis of accessory urethral was entertained. He was observed in the ward for five days and during all this time there were no events. The patient was reviewed at six weeks and three months post operation and found to be ok with no more discharge and the wound had healed well (Figure 9).The histology of the removed sinus tract was lined by stratified squamous epithelium (Figure10).

\section{Discussion}

Urethral duplication is a rare congenital anomaly with a variable clinical presentation that range from asymptomatic, obstructive symptoms, urinary incontinence, double stream, passage of urine in the perineum or rectally, epididymitis or recurrent urinary tract infections $[1,2,3]$. The anomaly may also be associated with other congenital anomalies such as anorectal malformations, renal agenesis, sacral agenesis, ectopic kidney, megaureter, unilateral or bilateral vesicoreteric reflux, hydronephrosis and cryptochirdism [1,4-8].

Therefore, it is very crucial to perform thoroughly physical examination and focused radiological test that include ultasonography of the urinary tract to rule out other congenital anomalies. Urethrogram and urethrocystoscopy may also be of paramount in assessing severity of the congenital anomaly [4].

The pathophysiology of urethral duplication remains obscure [2]. The proposed theories include a partial failure or an irregularity of the ingrowth of the lateral mesoderm between the ectodermal and endodermal layers of the cloaca membrane in the midline which explain the forms with a dorsal epispadiac channel by Casselman and Williams, abnormal termination of the mullerian duct by Das and Brosman and asymmetry in the closure of the urorectal septum by Rica et al [9-11]. However, none of the theories can explain all varieties of urethral duplication.

The widely accepted classification of urethral duplication is Effman's [11] classification (figure 11). Type I is the most common type where there is partial duplication of the urethra and is almost always asymptomatic, requiring no treatment [6]. Type II there is a complete duplication of the urethra and can be subdivided further into type IIA1 if both urethras arise from the separate bladder necks, type IIA2 (Y-type duplication) if one channel arises from the other, and type IIB if duplication with one meatus is observed $[12,13]$. Type III represents complete duplication of both urethra and bladder $[12,13]$ 


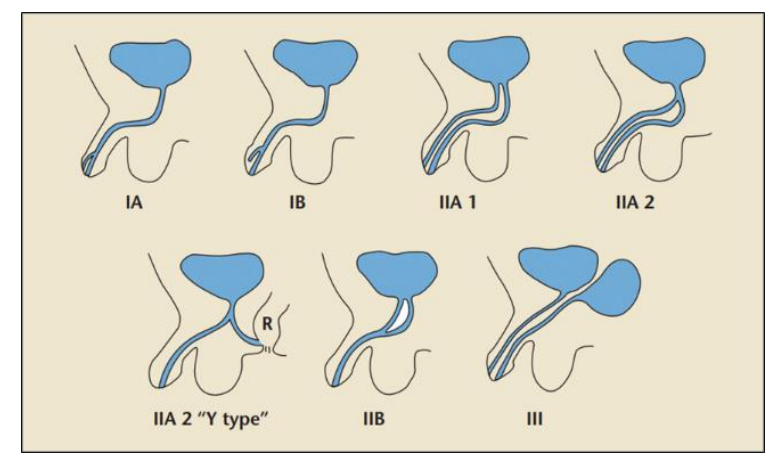

Figure 11 Effmann Classification for urethral duplication.

Our case was type IIA 2 "Y type" urethral duplication [13]. Our case presented relatively very late at the age of 19 years with pus discharge through small opening on the scrotum that was misdiagnosed initially as pilonidal sinus ( figure 11). In many cases of Y type urethral duplication, the accessory urethra opens in the perineum or rectum [12]. However, in our case the accessory urethra was ending at proximal bulbar urethra as shown in figure 7 above. Treatment of urethra duplication is individualized and depends upon anatomy of duplication and clinical presentation [1]. In our case, surgical removal of accessory urethra was performed because it was discharging pus. The patient had uneventful recovery three months postoperative.

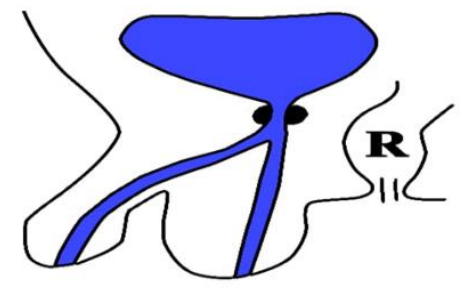

\section{IIA “' ${ }^{3 "}$}

Figure 12 Urethral duplication in our case in 19 years old boy.

\section{Conclusion}

Possibility of urethral duplication must be considered in any child or young adult presenting with scrotal sinus discharge before planning treatment. Treatment should always be individualized according to severity of symptoms and clinical presentation.

\section{Compliance with ethical standards}

\section{Acknowledgments}

We thank our patient for allowing us to publish this case report. We also extend our sincere gratitude to Heavenlight Luzinge for helping us with arranging photos and images in this manuscript.

\section{Disclosure of conflict of interest}

All authors listed in this case report declare that there is no conflict of interests of any kind.

\section{Statement of ethical approval}

The ethical clearance for this case report was obtained from Kilimanjaro Christian Medical University Research and Ethical Review Committee. 


\section{Statement of informed consent}

Written informed consent was obtained from the patient for publication of this case report and accompanying images.

\section{References}

[1] Haleblian G, Kraklau D, Wilcox D et al. Y-type urethral duplication in the male. BJUIInt. 2006; 97(3): 597-602.

[2] Erdil H, Mavi A, Erdil S, Gumusburun E. Urethral duplication. Acta Med Okayama. 2003; 57: 91-93.

[3] Mori K, Shin T, Tobu et al. A Case of Urethral Duplication Arising from the Posterior Urethra to the Scrotum with Urinary Stone in a 6-Year-Old Male. Hindawi Publishing Corporation Case Reports in Pediatrics. Volume 2014, Article ID 290623, 3 pages http://dx.doi.org/10.1155/2014/290623.

[4] Suoub M, Saleem MM, Sawaqed F. Complete Urethral Duplication: Case Report and Literature Review. Research and Reports in Urology. 2020; 12.

[5] Durrani KM, Shah PI, Kakalia GR. Interurethral fenestration for a case of double urethra with hypospadias. J Urol. 1972; 108: 586-590.

[6] Susan LP, Roth RB, Kaminsky AF. Complete duplication of urethra. Urology. 1975; 05: 390-393.

[7] Mehan DJ, Gonzales JH. Urethral duplication, with associated agenesis of left kidney and right ureteral ectopia. Urology. 1975; 6: 476-479.

[8] Naparstek S, Abrams HJ, Sutton AP, Buchbinder MI. Compete duplication of male urethra in children. Urology. 1980; 16: 391-392.

[9] Casselman J, Williams DI. Duplication of the urethra. Acta Urol Belg. 1996; 34: 535-541.

[10] Das S, Brosman SA. Duplication of the male urethra. J Urol. 1977; 117: 452-454.

[11] Effman EL, Lebowitz RL, Colodyn AH. Duplication of the urethra. Radiology. 1976; 119: 179-185.

[12] Rice PE, Holder TM and Ashcraft KW: Congenital posterior urethral perineal fistula: A case report. J Urol. 1978; 199: 416-417. 